\title{
Simple sequence repeat markers that identify Claviceps species and strains
}

\author{
Barbara S. Gilmore', Stephen C. Alderman', Brian J. Knaus², Nahla V. Bassil', Ruth C. Martin', \\ James E. Dombrowski ${ }^{*}$ and Jeremiah K. S. Dung ${ }^{4}$
}

\begin{abstract}
Background: Claviceps purpurea is a pathogen that infects most members of Pooideae, a subfamily of Poaceae, and causes ergot, a floral disease in which the ovary is replaced with a sclerotium. When the ergot body is accidently consumed by either man or animal in high enough quantities, there is extreme pain, limb loss and sometimes death.

Results: This study was initiated to develop simple sequence repeat (SSRs) markers for rapid identification of C. purpurea. SSRs were designed from sequence data stored at the National Center for Biotechnology Information database. The study consisted of 74 ergot isolates, from four different host species, Lolium perenne, Poa pratensis, Bromus inermis, and Secale cereale plus three additional Claviceps species, C. pusilla, C. paspali and C. fusiformis. Samples were collected from six different counties in Oregon and Washington over a 5-year period. Thirty-four SSR markers were selected, which enabled the differentiation of each isolate from one another based solely on their molecular fingerprints. Discriminant analysis of principle components was used to identify four isolate groups, CA Group 1, 2, 3, and 4, for subsequent cluster and molecular variance analyses. CA Group 1 consisting of eight isolates from the host species $P$. pratensis, was separated on the cluster analysis plot from the remaining three groups and this group was later identified as C. humidiphila. The other three groups were distinct from one another, but closely related. These three groups contained samples from all four of the host species. These SSRs are simple to use, reliable and allowed clear differentiation of C. humidiphila from C. purpurea. Isolates from the three separate species, C. pusilla, C. paspali and C. fusiformis, also amplified with these markers.
\end{abstract}

Conclusions: The SSR markers developed in this study will be helpful in defining the population structure and genetics of Claviceps strains. They will also provide valuable tools for plant breeders needing to identify resistance in crops or for researchers examining fungal movements across environments.

Keywords: Claviceps purpurea, Claviceps humidiphila, ERGOT, Simple sequence repeat primers, Perennial ryegrass, Kentucky bluegrass

\section{Background}

Mild winters and warm, dry summers in the Pacific Northwest of North America, where over $60 \%$ of the world's cool season grass seed is produced [1], are ideal for grass seed production. In Oregon, perennial ryegrass seed, Lolium perenne, and Kentucky bluegrass seed, Poa pratensis, are important crops. In 2014, 85 million kilograms of perennial ryegrass seed were produced, with a

\footnotetext{
*Correspondence: jim.dombrowski@ars.usda.gov

1 USDA ARS Forage Seed and Cereal Research, 3450 SW Campus Way,

Corvallis, OR 97331, USA

Full list of author information is available at the end of the article
}

farm gate value of US\$ 158 million [2]. Kentucky bluegrass seed produced in 2014 was over 8 million kilograms and valued at almost US\$ 23 million [2]. Claviceps species are important pathogens in the Pacific Northwest, where Claviceps infection can reduce yields in Kentucky bluegrass by as much as $47 \%$ by weight [3]. The genus Claviceps includes about 45 species [4] characterized by sclerotia which germinate to produce stalked, spherical fruiting bodies (capitula) embedded with perithecia. Ascospores produced within and ejected from the perithecia infect ovaries of grasses and sedges, transforming each infected ovary into an elongated or spherical 
sclerotium, depending on the Claviceps species. The best known species is $C$. purpurea which infects hundreds of grasses, primarily in the Pooideae subfamily of the grass family, including wheat, rye, and barley [5]. Alkaloids produced in the sclerotia are toxic, and if ingested by animals or humans result in ergotism. Ergotism is a condition characterized by constriction of the blood vessels, and can give rise to abortion, gangrenous limbs, and death from continued ingestion of the alkaloids [5].

Sclerotia of C. purpurea are black to purple-black and often extend beyond the host lemma and palea. Size of the sclerotium is proportionate to host seed size, with sclerotium size increasing with size of the host seed [5]. In addition to ascospores, conidia are produced during the early stages of infection and can contribute to secondary spread of the pathogen. Conidia mix with plant sap and ooze from infected florets in what is commonly referred to as the honeydew stage of disease development [5].

Breeding for resistance to $C$. purpurea or $C$. humidiphila requires an efficient means of identifying Claviceps strains, and improved knowledge of resistance genes in crops such as perennial ryegrass and Kentucky bluegrass [6-8]. The ability to fingerprint $C$. purpurea isolates would allow identification of more virulent strains and assist plant breeders in the evaluation of the resistance genes that are available in the different cereal or grass gene pools. In 1997, Jungehülsing and Tudzynski determined that non-rye ergot isolates were less aggressive on rye than isolates from rye [9]. Pažoutová [10] also concluded that the genetic resistance of host grasses may vary with the C. purpurea isolate used for evaluation. Therefore, the isolate strains should originate from locations that are similar to those intended for growth of the resistant cultivar [10]. This suggests that an appropriate isolate-host pair is required for identifying resistance in host crops. Isolate identification would also allow monitoring fungal movement in terms of both distance and speed across previously non-infected fields. SSR markers would then prove to be a valuable molecular tool for differentiating Claviceps species and strains for breeding programs and for gaining a better understanding of Claviceps population biology and genetics.

Previously, sixteen Randomly Amplified Polymorphic DNA (RAPD) primer pairs were successfully used to discriminate between 29 field isolates of $C$. purpurea from various parts of Europe [9]. In addition to RAPDs, alkaloid analysis and conidial measurements were also used to discriminate C. purpurea isolated from or found growing in three diverse environments: open meadows and fields, shady or wet grassy areas, and salt marshes [11, 12]. These three populations were originally designated as genotypes G1, G2 and G3 respectively but are now recognized as $C$. purpurea sensu stricto, $C$. humidiphila, and C. spartinae, respectively. The sclerotia of the three species were found to produce different sets of alkaloids; C. purpurea sensu stricto sclerotia contains ergotamine, ergosine, ergocornine, ergocryptine and ergocristine; C. humidiphila produces ergosine, ergocristine and ergocryptine; and C. spartinae produced ergocristine and ergocryptine [11, 13]. In 2015, a new genetic group was added, G2a (G4), C. arundinis, which inhabited hosts found growing in very wet areas and produced sclerotia that contained the alkaloids, ergosine, ergocristine, ergocristam and ergosedmam $[13,14]$.

Amplified Fragment Length Polymorphism (AFLP) marker patterns and EcoRI restriction site polymorphism in the 5.85S ribosomal DNA (rDNA) have also been used $[11,15]$. Based on RAPD and AFLP analyses of Spartina alterniflora ergot, G1, G2, and G3 isolates were each present in a specific geographical area, but G3 had little variation, possibly indicating recent introduction [15]. Phylogenetic analyses of $C$. purpurea populations using DNA sequences from an internal transcribed spacer region (ITS) and a portion of the gene encoding $\beta$-tubulin indicated that the G1 types had diverted significantly from the G2/G3 types [16]. According to Pažoutová [11], these populations can only be differentiated by molecular methods, and are not phenotypically, host or habitat distinguishable, but do appear to be habitat-specialized.

Additional molecular resources for C. purpurea are available and include expressed sequence tags (ESTs) [17], and a genome sequence, based on Roche/454 chemistry [18]. While informative techniques have been used to differentiate $C$. purpurea isolates, SSRs were not developed despite their reported value as co-dominant, multi-allelic, abundant, easily implemented, and highly reproducible markers [19]. Existing sequence resources can be applied for the development of additional SSR markers in a genus like Claviceps that has few available SSRs. Given the importance of identifying different isolates, the objective of this study was to develop SSR markers to facilitate differentiation of Claviceps purpurea isolates and its closely related species.

\section{Results and discussion}

Twenty-five of the 74 isolates used in this study were previously characterized by RAPD patterns, ITS sequences and mating type PCR assays by Scott et al. [20] and identified as C. purpurea, (G1), isolated from L. perenne and P. pratensis, and C. humidiphila (G2), from P. pratensis. Only molecular methods were used to distinguish these isolates from each other, since they were collected from similar environments. The SSRs developed in this study were evaluated in the 74 isolates. 


\section{Simple sequence repeats (SSRs)}

The microsatellites were mined from 12 assembled genomic sequences from data stored at NCBI [21]. Primers were designed for 267 SSR sequences but only the first 192 primer pairs were evaluated in this study. In the first screening, 123 of the 192 primers tested in four isolates (Cp03, Cp26, Cp32 and Cp33) (Table 1) appeared polymorphic, 20 had missing data or null alleles, 17 were monomorphic and 32 failed to amplify. Eight isolates (Cp25, Cp26, Cp27, Cp29, Cp30, Cp31, Cp32, and Cp33) (Table 1) were used in the second screening to evaluate 59 primer pairs that were selected based on polymorphism in the first screening and ease of multiplexing. The criteria for primer selection of the resulting fingerprinting panel were ease of scoring, high polymorphism and amplicon sizes that permitted multiplexing. The results from the second screening yielded 34 primer pairs that were placed into seven multiplex pools post PCR amplification for capillary electrophoresis separation.

\section{Fingerprinting and genetic diversity evaluation}

The motifs for the 34 SSRs included two dinucleotides (5.9\%), 22 trinucleotides (64.7\%), six tetranucleotides $(17.6 \%)$, and four pentanucleotides (11.7\%) (Table 2). The number of alleles in the 77 evaluated isolates varied from a low of two alleles for primers Cpur20 and Cpur72 to a high of 15 alleles for Cpur56, and averaged 5.8 alleles per primer pair (Table 2). Twenty-one SSR primer pairs generated one allele per sample, while 12 primer pairs yielded up to two alleles for some or all samples, and one primer pair, Cpur69, amplified three alleles in one individual, 14-040 (Table 2).

The average genetic distance between samples of $C$. fusiformis, C. paspali, C. pusilla and the remaining samples was $0.784,0.691$, and 0.767 , respectively. These relatively large genetic distances indicated that samples from these three species, C. fusiformis, C. paspali and C. pusilla, were not closely related to the other samples. Furthermore, these species had a large number of unique alleles at many tested SSRs that were not shared with samples from the other species, supporting their distinctness (Table 1). Consequently, these three species were not included in subsequent statistical analyses.

\section{Statistical analysis}

In order to infer and distinguish an optimal number of groups within the isolates evaluated, discriminant analysis of principle components was performed. K-means clustering was performed on 40 principle components derived from the data. For group sizes ranging from 1 to 40 groups, we examined the Bayesian information criteria (BIC) for each grouping as a function of the number of clusters, and concluded that a group number of four minimized the BIC. These four clusters were referred to with the CA prefix for cluster analysis to indicate the groups that were selected for subsequent cluster and molecular variance analyses. CA Group 1 was comprised of samples collected from the host, $P$. pratensis, in Washington and Oregon. These samples were identified as $C$. humidiphila by Scott et al. [20]. CA Group 2 consisted primarily of eight samples from the $P$. pratensis host, but also contained two samples from Bromus inermis and five from L. perenne. CA Group 3 and CA Group 4 were solely made up of samples from $L$. perenne, except for a single sample of $S$. cereale that was found in CA Group 3 (Table 1).

The shared allele distances were estimates of genetic distances. The average genetic distance of CA Group 1 to the remaining 66 samples was large at 0.915 . The average genetic distance for CA Group 2, Group 3 and, Group 4 were intermediate at $0.522,0.502$, and 0.495 , respectively. These values supported the K-means cluster analysis results (Fig. 1). It is interesting to note that most of the Oregon samples isolated from the $P$. pratensis host were not far removed from the isolates found on the L. perenne host.

The CA Group 1 samples were monomorphic at 16 of the 34 SSRs, but polymorphic in the remaining 18 SSRs (Table 2). However, 13 of the 34 SSRs (Cpur07, Cpur08, Cpur12, Cpur14, Cpur23, Cpur24, Cpur30, Cpur31, Cpur35, Cpur69, Cpur72, Cpur145, and Cpur157) contained CA Group 1-specific alleles from P. pratensis grown in Washington that separated isolates of this group from those of the remaining three CA groups. Samples from CA Group 2, 3, and 4 were more diverse than those from Group 1 and had fewer monomorphic SSRs at 4, 5, and 6 SSRs, respectively.

Surprisingly, samples from CA Groups 3 and 4 separated into two clusters despite sharing the same host, and similar geographical origin in two counties in Oregon. All five collection years, 2010 through 2014, were represented in both groups, as were most of the cultivars (Table 1). Upon further examination of the SSR data we found that samples from these two groups had distinct alleles at seven SSRs: Cpur2, Cpur8, Cpur30, Cpur31, Cpur32, Cpur34, and Cpur35. Cluster analysis based on data for these 7 markers separated the samples into two groups. CA Group 3 and CA Group 4 did not intermingle except for one sample of CA Group 3 (Cp38) that grouped with the samples from CA Group 4 (not shown). These seven SSRs can therefore be used for distinguishing samples from each of these two groups isolated from very similar environments and hosts (Table 2).

Diversity estimates calculated at each SSR in these 74 samples consisted of the number of alleles per primer pair $(A)$ and Shannon-Wiener's index coefficient $(H)$ 
Table 1 List of 74 isolates of Claviceps purpurea (Cp) and one each of $C$. pusilla, C. paspali and C. fusiformis

\begin{tabular}{|c|c|c|c|c|c|c|}
\hline Sample & Host & Year & Cultivar & State & Cty & CA Group \\
\hline C. purpurea, Cp01 & L.perenne & 2012 & Pavilion & OR & Um & 2 \\
\hline C. purpurea, Cp02 & L.perenne & 2012 & Pavilion & OR & Um & 3 \\
\hline C. purpurea, $\mathrm{Cp}_{0} 3^{\mathrm{a}}$ & L.perenne & 2012 & Pavilion & OR & Um & 3 \\
\hline C. purpurea, Cp04 & L.perenne & 2012 & Provocative & OR & Um & 2 \\
\hline C. purpurea, Cp05 & L.perenne & 2012 & Provocative & OR & Um & 2 \\
\hline C. purpurea, Cp06 & L.perenne & 2012 & Top Hat II & OR & Um & 4 \\
\hline C. purpurea, Cp07 & L.perenne & 2012 & Top Hat II & OR & Um & 3 \\
\hline C. purpurea, Cp08 & L.perenne & 2012 & Pavilion & OR & Um & 2 \\
\hline C. purpurea, Cp10 & L.perenne & 2012 & Pavilion & OR & Um & 3 \\
\hline C. purpurea, Cp11 & L.perenne & 2011 & Esquire & OR & Um & 3 \\
\hline C. purpurea, Cp12 & L.perenne & 2011 & Esquire & OR & Um & 3 \\
\hline C. purpurea, Cp13 & L.perenne & 2011 & Esquire & OR & Um & 3 \\
\hline C. purpurea, Cp14 & P. pratensis & 2012 & Midnight & WA & $\mathrm{Be}$ & 1 \\
\hline C. purpurea, Cp15 & P. pratensis & 2012 & Midnight & WA & $\mathrm{Be}$ & 1 \\
\hline C. purpurea, Cp16 & P. pratensis & 2012 & Midnight & WA & $\mathrm{Be}$ & 1 \\
\hline C. purpurea, Cp20 & P. pratensis & 2012 & Seed screenings & WA & $\mathrm{Be}$ & 1 \\
\hline C. purpurea, Cp21 & P. pratensis & 2012 & Seed screenings & WA & $\mathrm{Be}$ & 1 \\
\hline C. purpurea, Cp22 & P. pratensis & 2012 & Arrowhead & WA & $\mathrm{Be}$ & 1 \\
\hline C. purpurea, Cp23 & P. pratensis & 2012 & Arrowhead & WA & $\mathrm{Be}$ & 1 \\
\hline C. purpurea, Cp25 b & S. cereale & 2012 & Wild & OR & Um & 3 \\
\hline C. purpurea, Cp26 $6^{a, b}$ & L.perenne & 2011 & Seed screenings & $\mathrm{OR}$ & Um & 3 \\
\hline C. purpurea, Cp27b & L.perenne & 2011 & Seed screenings & OR & Um & 4 \\
\hline C. purpurea, Cp29 & L.perenne & 2012 & Esquire & OR & Um & 4 \\
\hline C. purpurea, Cp30 & B. inermis & 2011 & Wild & WA & Wh & 2 \\
\hline C. purpurea, Cp31 b & B. inermis & 2011 & Wild & WA & Wh & 2 \\
\hline C. purpurea, Cp32 & P. pratensis & 2013 & Seed screenings & OR & Un & 1 \\
\hline C. purpurea, Cp33 & P. pratensis & 2013 & Baron & OR & Un & 2 \\
\hline C. purpurea, Cp34 & L.perenne & 2013 & Provocative & OR & Um & 3 \\
\hline C. purpurea, Cp35 & L.perenne & 2013 & Provocative & OR & Um & 3 \\
\hline C. purpurea, Cp37 & L.perenne & 2013 & Zoom & OR & Um & 3 \\
\hline C. purpurea, Cp38 & L.perenne & 2013 & Zoom & OR & Um & 3 \\
\hline C. purpurea, Cp39 & L.perenne & 2013 & Zoom & OR & Um & 2 \\
\hline C. purpurea, Cp40 & L.perenne & 2014 & & OR & Um & 4 \\
\hline C. purpurea, Cp41 & L.perenne & 2014 & & OR & Um & 3 \\
\hline C. purpurea, Cp42 & L.perenne & 2014 & & OR & Um & 3 \\
\hline C. purpurea, Cp43 & L.perenne & 2014 & Casper & OR & Um & 3 \\
\hline C. purpurea, Cp44 & L.perenne & 2014 & Casper & OR & Um & 4 \\
\hline C. purpurea, Cp45 & L.perenne & 2014 & Casper & OR & Um & 4 \\
\hline C. purpurea, Cp46 & L.perenne & 2014 & Esquire & OR & Um & 4 \\
\hline C. purpurea, Cp47 & L.perenne & 2014 & Esquire & OR & Um & 4 \\
\hline C. purpurea, Cp48 & L.perenne & 2014 & Esquire & OR & Um & 4 \\
\hline C. purpurea, Cp49 & L.perenne & 2014 & Frontier & OR & Um & 3 \\
\hline C. purpurea, Cp50 & L.perenne & 2014 & Frontier & OR & Um & 3 \\
\hline C. purpurea, Cp51 & L.perenne & 2014 & Frontier & OR & Um & 4 \\
\hline C. purpurea, Cp52 & P. pratensis & 2010 & Seed screenings & OR & Je & 2 \\
\hline C. purpurea, Cp53 & P. pratensis & 2010 & Seed screenings & OR & Je & 2 \\
\hline C. purpurea, Cp54 & P. pratensis & 2010 & Seed screenings & OR & Je & 2 \\
\hline C. purpurea, Cp55 & L.perenne & 2013 & Esquire & OR & $\mathrm{Be}$ & 4 \\
\hline C. purpurea, Cp56 & L.perenne & 2013 & Esquire & OR & $\mathrm{Be}$ & 3 \\
\hline
\end{tabular}


Table 1 continued

\begin{tabular}{|c|c|c|c|c|c|c|}
\hline Sample & Host & Year & Cultivar & State & Cty & CA Group \\
\hline C. purpurea, Cp57 & L.perenne & 2013 & Esquire & OR & $\mathrm{Be}$ & 4 \\
\hline C. purpurea, Cp58 & L.perenne & 2014 & & OR & Um & 3 \\
\hline C. purpurea, Cp59 & L.perenne & 2014 & & OR & Um & 4 \\
\hline C. purpurea, Cp61 & L.perenne & 2013 & Zoom & OR & Um & 3 \\
\hline C. purpurea, Cp62 & L.perenne & 2013 & Zoom & OR & Um & 3 \\
\hline C. purpurea, Cp63 & L.perenne & 2013 & Zoom & OR & Um & 3 \\
\hline C. purpurea, Cp64 & L.perenne & 2013 & PST-2M20 & OR & $\mathrm{Be}$ & 4 \\
\hline C. purpurea, Cp65 & P. pratensis & 2012 & Baron & OR & Un & 2 \\
\hline C. purpurea, Cp66 & L.perenne & 2012 & seed screenings & OR & Um & 4 \\
\hline C. purpurea, Cp67 & L.perenne & 2012 & seed screenings & OR & Um & 4 \\
\hline C. purpurea, Cp69 & L.perenne & 2014 & Pavillion & OR & Um & 4 \\
\hline C. purpurea, Cp70 & L.perenne & 2014 & Pavillion & OR & Um & 3 \\
\hline C. purpurea, Cp71 & L.perenne & 2014 & Pavillion & $\mathrm{OR}$ & Um & 3 \\
\hline C. purpurea, Cp72 & L.perenne & 2013 & Provocative & OR & Um & 4 \\
\hline C. purpurea, Cp73 & L.perenne & 2013 & Provocative & OR & Um & 3 \\
\hline C. purpurea, Cp74 & L.perenne & 2013 & Provocative & OR & Um & 3 \\
\hline C. purpurea, Cp78 & P. pratensis & 2012 & Baron & OR & Un & 2 \\
\hline C. purpurea, Cp79 & P. pratensis & 2012 & Baron & OR & Un & 2 \\
\hline C. purpurea, Cp80 & P. pratensis & 2012 & Baron & OR & Un & 2 \\
\hline C. purpurea, 14-036-F & L.perenne & 2013 & Pavillion & OR & Um & 4 \\
\hline C. purpurea, 14-037-F & L.perenne & 2013 & Pavillion & OR & Um & 4 \\
\hline C. purpurea, 14-038-F & L.perenne & 2013 & Pavillion & OR & Um & 4 \\
\hline C. purpurea, 14-039-F & L.perenne & 2013 & Pavillion & OR & Um & 4 \\
\hline C. purpurea, 14-040-F & L.perenne & 2013 & Pavillion & OR & Um & 4 \\
\hline C. purpurea, 14-041-F & L.perenne & 2013 & Pavillion & OR & Um & 3 \\
\hline \multicolumn{7}{|l|}{ C.pusilla } \\
\hline \multicolumn{7}{|l|}{ C.paspali } \\
\hline C. fusiformis & & & & & & \\
\hline
\end{tabular}

Host plant species, year of collection, name of host cultivar, state where collected, county (Cty) and cluster analysis (CA) Group are provided

ST State where sample was collected, OR (Oregon), WA (Washington)

Cty County where sample was collected, Be (Benton), Je (Jefferson), Um (Umatilla), Un (Union), and Wh (Whitman)

a Four samples used for the first screening

b Eight samples used for the second screening

(Table 2). The number of alleles per primer pair ranged from a low of two at Cpur20 and Cpur72 to a high of 15 alleles at Cpur56 and averaged 5.75. This was higher than expected since many fungi are very homogeneous with little polymorphism [22]. The coefficient $H$ was used to measure the allelic diversity at each locus. Due to the low number of alleles at each SSR Shannon-Wiener's index coefficient $(H)$ values were low for most of the primer pairs and ranged from 0.34 at primer Cpur72 to a high of 1.85 at Cpur56 with an average of 1.09.

Sample size was more balanced in the four CA Groups than in the plant hosts $P$. pratensis, L. perenne, B. inermis and S. cereale. Therefore, the Analysis of Molecular Variance, AMOVA, (Table 3), was based on the four CA Groups using $147(\mathrm{n}-1)$ degrees of freedom $(d f)$ to code the data as diploid in the 74 samples. Where a single allele was amplified, the sample was considered homozygous at this locus. The AMOVA confirmed that the molecular variance between the samples in the groups, $56.7 \%$, was higher than the variance between the CA Groups, $41.5 \%$ (Table 3).

As illustrated in the dendrogram (Fig. 2), each of the 74 samples produced a unique fingerprint. Samples from the Washington $P$. pratensis CA Group 1 were isolated from all other branches of the dendrogram with a high bootstrap value of $100 \%$. High bootstrap support of $93 \%$ was observed in only Cp05 and Cp04 of CA Group 2d samples consisting of $P$. pratensis, B. inermis, and L. perenne, from Oregon. The remaining isolates of CA Group 2 were found in three branches, CA Group 2a, Group 2b and 


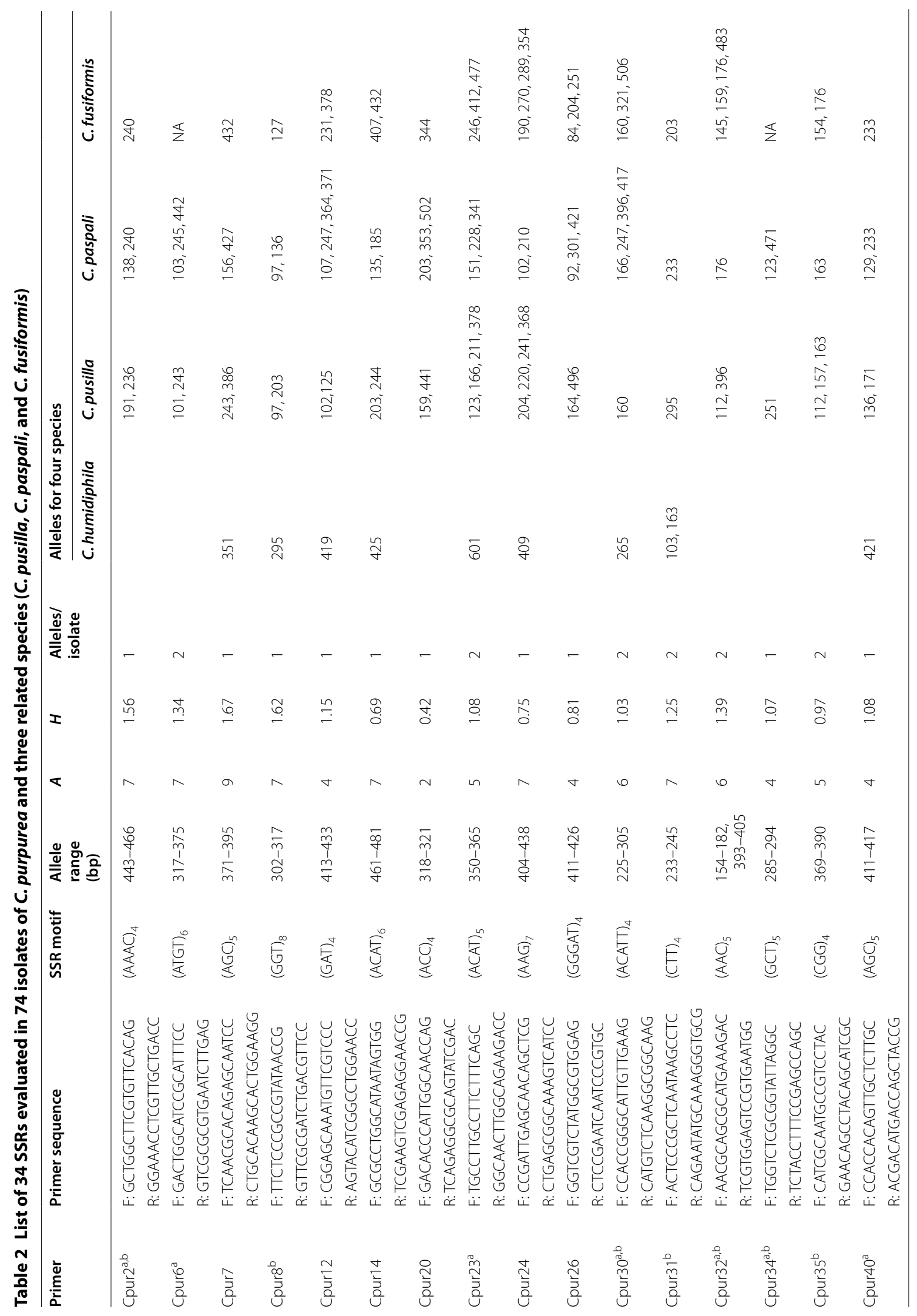




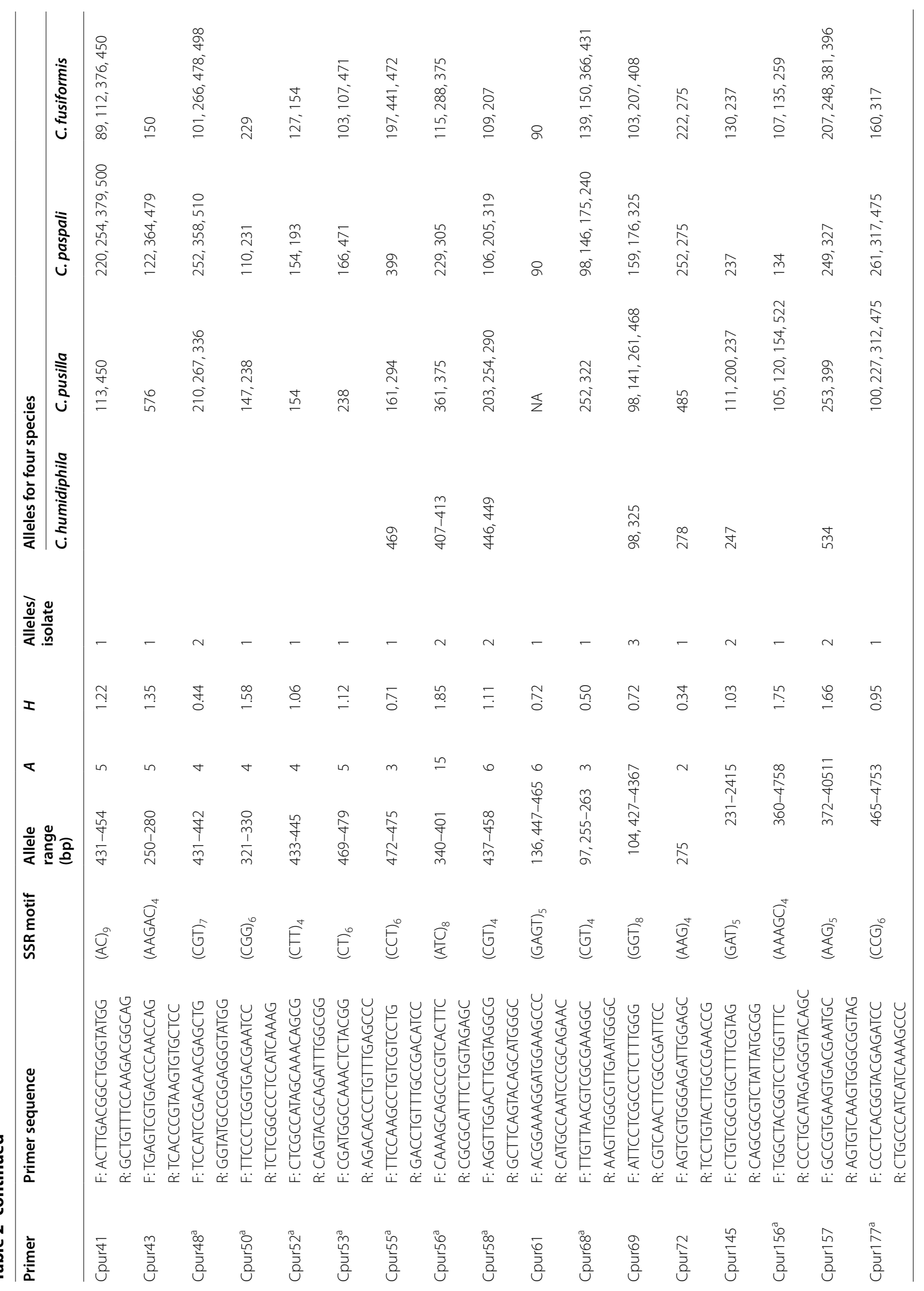




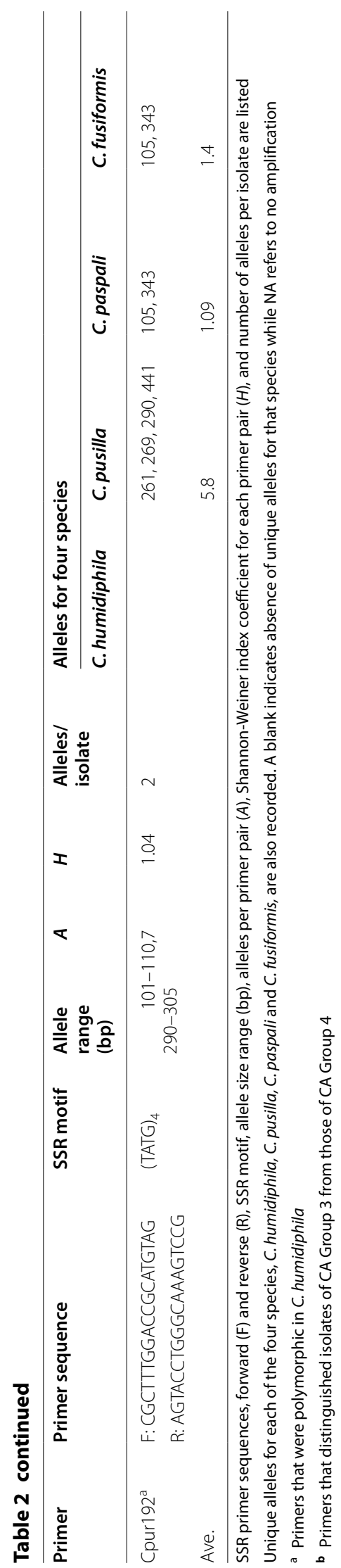




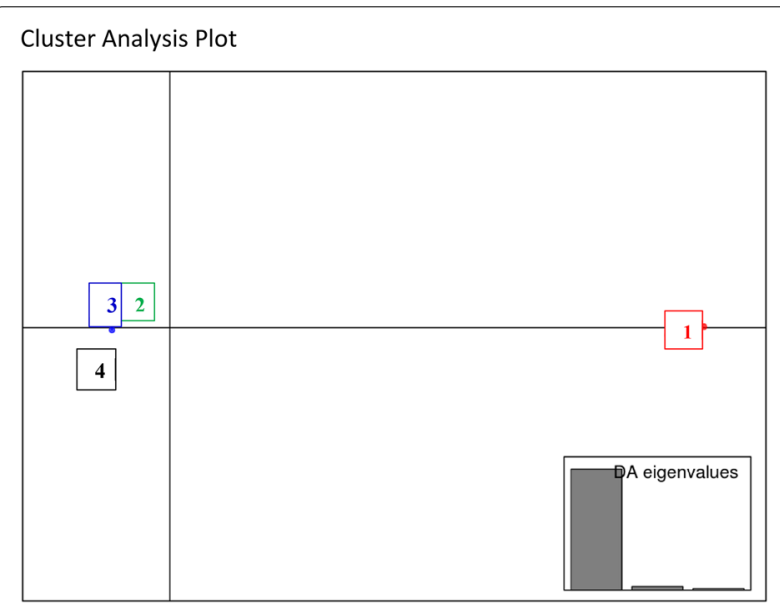

Fig. 1 Cluster analysis plot. A plot showing the spatial relationship of our four designated CA Groups. CA Group 1 is C. humidiphila and CA Groups 3 and 4 are C. purpurea

Group 2c. Isolates of CA Group 3 clustered mainly into two main branches, CA Group 2a and Group 3b, except for $\mathrm{Cp} 25$ from $S$. cereale that was grouped with CA Group 2b samples. A small number of isolates from CA Group 4 clustered together with high bootstrap support (86\%) in CA Group 4a while the majority were grouped together into one branch, CA Group 4b (Fig. 2).

\section{Conclusion}

Using 34 microsatellites, we identified each of the 74 isolates of $C$. purpurea collected from four hosts and separated them into four separate groups. CA Group 1 isolates contained all the isolates from the P. pratensis host from Washington and were widely separated from isolates in the remaining three groups. Based on Scott et al. [20], this group represents C. humidiphila. Isolates from the same $P$. pratensis host but from the state of Oregon however grouped separately into CA Group 2 with $C$. purpurea samples from $B$. inermis and $L$. perenne. More work needs to be completed to evaluate the relationship between isolates of $P$. pratensis from Oregon that were in CA Group 2 and those ' $C$. humidiphila' isolates of CA Group 1 to determine if these two groups are both $C$. humidiphila, or if only CA Group 1 is that species.

Claviceps purpurea in both CA Group 3 and CA Group 4 were isolated from a single host species, L. perenne, and from the same geographical regions. These SSR markers easily separated the isolates into two groups, possibly indicating distinct populations. These results support the finding of Pažoutová et al. [11] that molecular are necessary to distinguish separate Claviceps populations. Multiple populations could arise through different seed sources, or contamination during seed harvest, storage, and cleaning. Contamination can also occur from susceptible weed grasses within fields. This indicates that proper isolate identification is critical in a breeding program aimed at developing resistance to this pathogen in a particular plant species. Repeated testing with the same isolates is necessary to identify effective resistance genes and can now be accomplished. The putative resistant genes can be tested against the same virulent strains, and resistance can then be introgressed into new cultivars. It will also be possible to follow $C$. purpurea and $C$. humidiphila movements through geographical areas.

In addition to distinguishing C. purpurea isolates, these SSRs also amplified in isolates from three other Claviceps species, C. pusilla, C. paspali and C. fusiformis, indicating possible usefulness in identifying isolates across this genus. Therefore, these markers may also be valuable for species identification.

\section{Methods}

\section{Fungal cultures and DNA extraction}

The 74 isolates used in this study were collected from $P$. pratensis and L. perenne in Oregon and Washington over a period of 5 years (2010-2014). We use the prefix Cp to refer to each $C$. purpurea isolate.

Sclerotia were surface sterilized by dipping in $95 \%$ ethanol for $30 \mathrm{~s}$, soaking in $0.6 \%$ sodium hypochlorite solution for $1 \mathrm{~min}$, and rinsing in sterile water for $15 \mathrm{~s}$. Sclerotia were bisected with a flame-sterilized blade and placed, cut surface down on water agar in a $9.5 \mathrm{~cm}$ diameter petri plate. Single hyphal tips were each transferred to potato dextrose agar to establish hyphal tip cultures.

Table 3 The AMOVA values

\begin{tabular}{lcccccc}
\hline Source of variation & $\boldsymbol{d f}$ & Sum of squares & Mean square & Expected mean square & \% Variance & $\boldsymbol{P}$ value \\
\hline Between groups & 3 & 408.218 & 136.073 & 3.61 & 41.5 & 0.001 \\
Between samples within groups & 70 & 701.762 & 10.025 & 4.93 & 56.71 & 0.001 \\
Within samples & 74 & 11.500 & 0.155 & 0.16 & 1.79 & 0.001 \\
Total & 147 & 1121.48 & 7.63 & 8.7 & 100 & 0.001 \\
\hline
\end{tabular}

The AMOVA table is based on an analysis of the groups which resulted from the cluster analysis. Presented p-values are based on one thousand permutations 


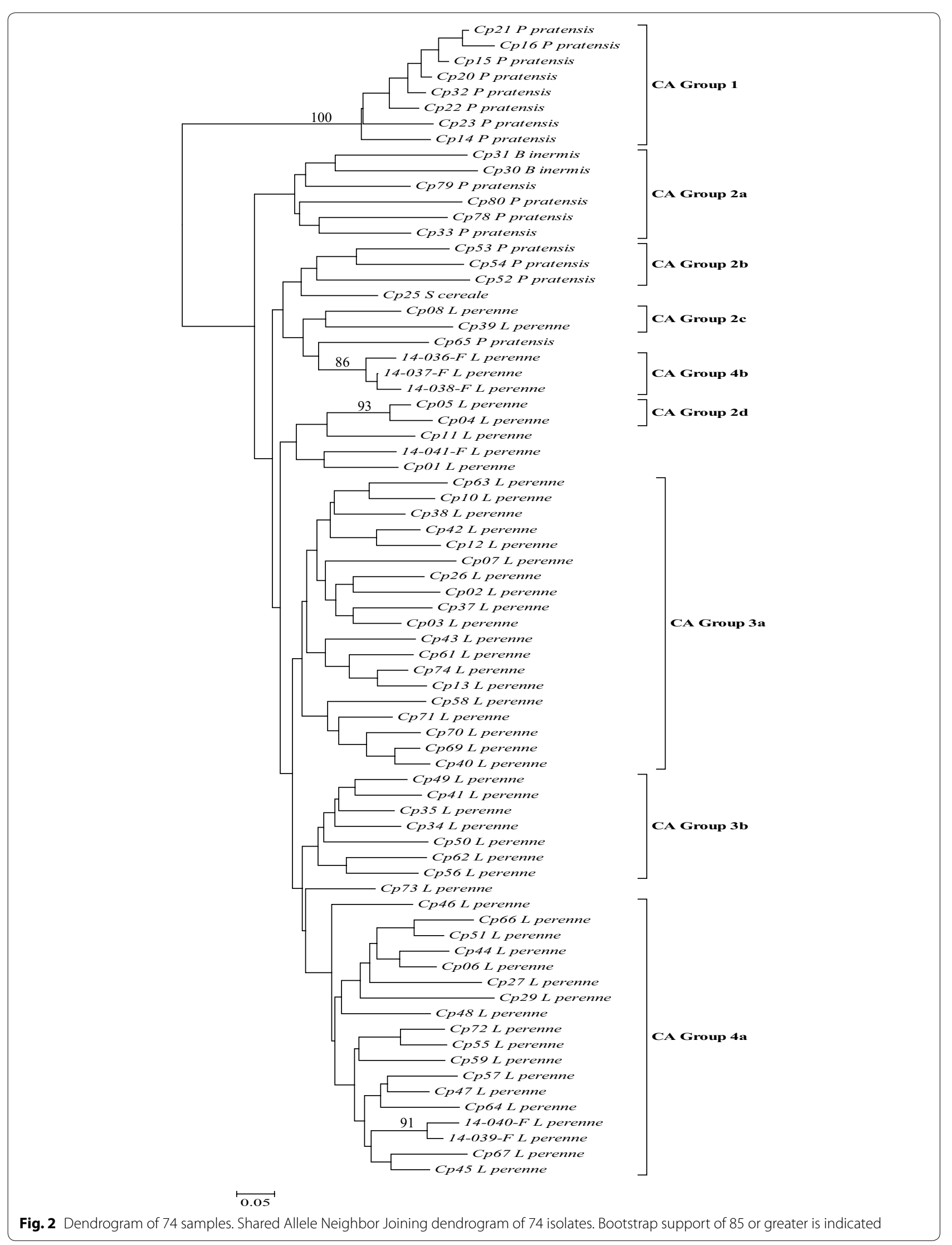


Cultures used for extraction were raised on a Claviceps medium, containing potato dextrose agar (PDA) $(18 \mathrm{~g})$, yeast extract $(1 \mathrm{~g})$, malt extract $(5 \mathrm{~g})$, sucrose $(5 \mathrm{~g})$, agar $(2.5 \mathrm{~g})$, and water $(500 \mathrm{ml})$. Cultures were grown at room temperature $\left(22{ }^{\circ} \mathrm{C}\right)$ for three weeks and then harvested for DNA extraction. The mycelial growth was scraped off of the agar, ground in liquid nitrogen using a mortar and pestle and the powder placed into two $2 \mathrm{ml}$ centrifuge tubes. Then Qiagen Cell Lysis Solution (Qiagen, Inc., Valencia, CA, Cat. No. 158908), along with RNAse A and Proteinase $K$ were added and the DNA was isolated with the Qiagen protocol as detailed in Gilmore et al. [23].

\section{Microsatellite marker development}

We named each SSR locus 'Cpur' for C. purpurea followed by a number indicating the specific sequence the SSR was designed from (Table 2). SSR primers for $C$. purpurea were designed from the short read genomic sequence data generated by Schardl et al. [18]. The contig sequence information for 192 contigs, generated with the Roche/454 Titanium sequencer, was downloaded from NCBI. Twelve assembled sequences were pasted into MSATCOMMANDER [24], which identified 267 SSR sequences while Primer3 designed primer pairs for each SSR [25-27]. Default settings for both programs were used except that trinucleotide, tetranucleotide and pentanucleotide repeats were also included on the SSR search. An M13 sequence (TGTAAAACGACGGCCAGT) was added to the $5^{\prime}$ end of the forward primers to allow for an economic method of fluorescent labeling of PCR products [28]. A PIG-tail sequence, 5'-GTTT$3^{\prime}$, was added to the $5^{\prime}$ end of the reverse primer, to promote full adenylation of fragments and reduce the number of split peaks [29]. A total of 192 M13-tagged forward primers and corresponding reverse primers, along with fluorescently labeled universal M13 (-21) forward primers (WellRED D2, D3, or D4) were ordered from Integrated DNA Technologies (IDT, San Diego, CA). The primers were tested for amplification in four isolates, Cp03, Cp26, Cp32 and Cp33. Fifty-nine primer pairs were identified as potential candidates for fingerprinting and were subjected to a second round of testing with eight isolates (Cp25, Cp26, Cp27, Cp29, Cp30, Cp31, Cp32, and Cp33). Thermocycler amplification of the M13-tagged SSRs was performed with a touchdown PCR, consisting of an initial denaturing step of $94{ }^{\circ} \mathrm{C}$ for $3 \mathrm{~m}$, then 10 cycles of $94{ }^{\circ} \mathrm{C}$ for $40 \mathrm{~s}, 62^{\circ} \mathrm{C}$ for $45 \mathrm{~s}$ (decreasing the annealing temperature by $1.0{ }^{\circ} \mathrm{C}$ per cycle), and $72{ }^{\circ} \mathrm{C}$ for $45 \mathrm{~s}$ followed by 20 cycles of $94{ }^{\circ} \mathrm{C}$ for $40 \mathrm{~s}, 52{ }^{\circ} \mathrm{C}$ for $45 \mathrm{~s}$, and $72{ }^{\circ} \mathrm{C}$ for $45 \mathrm{~s}$; eight cycles of $94{ }^{\circ} \mathrm{C}$ for $40 \mathrm{~s}, 53{ }^{\circ} \mathrm{C}$ for $45 \mathrm{~s}$, and $72{ }^{\circ} \mathrm{C}$ for $45 \mathrm{~s}$; and a final extension of $72{ }^{\circ} \mathrm{C}$ for $30 \mathrm{~min}$. The $15-\mu \mathrm{L}$ PCR reaction mix contained: $3 \mu \mathrm{L}$ of $5 \mathrm{X}$ GoTaq DNA Polymerase Buffer (Promega Corp., Madison, WI); $1.2 \mu \mathrm{L}$ of $2.5 \mathrm{mM}$ dNTPs; $1.2 \mu \mathrm{L}$ of $25 \mathrm{mM} \mathrm{MgCl}_{2} ; 0.075 \mu \mathrm{L}$ of 5 $\mathrm{U} / \mu \mathrm{L}$ GoTaq DNA Polymerase; $0.18 \mu \mathrm{L}$ of $10 \mathrm{mM}$ forward primer; $0.75 \mu \mathrm{L}$ of $10 \mathrm{mM}$ reverse primer; 0.75 $\mu \mathrm{L}$ of $10 \mathrm{mM}$ M13-fluorescent tag, WellRED D2, D3, or D4; and $1.5 \mu \mathrm{L}$ of $3 \mathrm{ng} \mu \mathrm{L}^{-1}$ template DNA. Since the expected size of each amplicon was not known, an equal number of primer pairs was labeled with the different dyes, WellRED D2, D3, and D4 to allow pooling for capillary electrophoresis separation using the Beckman Coulter CEQ 8000 (Beckman Coulter, Inc., Brea CA). PCR products amplified in this first screening in the four test genotypes were then separated by $1.5 \%$ agarose gel electrophoresis (1X TBE), and visualized with ethidium bromide to confirm amplification. PCR products from three primer pairs labeled with each of the three dyes were pooled and separated by capillary electrophoresis. The electropherograms were then scored and multiplexes were developed based on amplification and lack of fragment overlap.

\section{Statistical analysis}

In order to infer and distinguish an optimal number of groups within the sample, discriminant analysis of principle components was performed using the $\mathrm{R}$ package adegenet [30-33]. The optimal number of groups was determined using the function adegenet::find.clusters. We retained 40 principle components and examined a plot of Bayesian information criteria (BIC) as a function of the number of clusters for each analysis and concluded that a group number of four minimized the BIC. These groups were then submitted to discriminant analysis using adegenet::dapc and visualized. Statistical significance was determined for these groups using the Poppr function poppr::poppr.amova [34] using one thousand replicates to determine significance. A neighbor-joining tree was created using the function poppr::bruvo. boot [35] [34] using one thousand bootstrap replicates. The dendrogram was visualized in MEGA4 [36]. PowerMarker was used to identify markers that had the greatest impact on forming CA Groups 3 and 4 and to calculate the shared allele genetic distance [37]. Shannon's-Wiener's index $(H)$ was calculated using the following formula [38].

$$
H=-\sum_{i=1}^{n} p i \ln p i
$$

\section{Authors' contributions}

BG conceived of the study, participated in the study design, performed data collection and analysis, participated in data interpretation, and participated in drafting the manuscript. SA conceived of the study, participated in the study design, provided funds for data collection, and participated in drafting 
the manuscript. BK participated in the study design, performed analysis and interpretation of data; and participated in drafting the manuscript. NB participated in collection, analysis and interpretation of data, provided facilities and equipment for data collection, and participated in drafting the manuscript. RM participated in the study design and in drafting the manuscript. JD contributed to interpretation of data and participated in drafting the manuscript. JD supplied isolate material for study, provided background information, and participated in drafting the manuscript. All authors read and approved the final manuscript.

\section{Author details}

${ }^{1}$ USDA ARS Forage Seed and Cereal Research, 3450 SW Campus Way, Corvallis, OR 97331, USA. ${ }^{2}$ USDA ARS Horticultural Crops Research Unit, 3420 NW Orchard Ave, Corvallis, OR 97331, USA. ${ }^{3}$ USDA ARS National Clonal Germplasm Repository, 33447 Peoria Road, Corvallis, OR 97333, USA. ${ }^{4}$ Oregon State University, Central Oregon Agricultural Research Center, 850 NW Dogwood Lane, Madras, OR 97741, USA.

\section{Acknowledgements}

We would like to thank Dr. Paul W. Tooley (USDA-ARS, Maryland) for donating tissue of C. pusilla, C. paspali and C. fusiformis for our research.

The use of trade, firm, or corporation names in this publication is for the information and convenience of the reader. Such use does not constitute an official endorsement or approval by the United States Department of Agriculture or the Agricultural Research Service of any product or service to the exclusion of others that may be suitable.

\section{Competing interests}

The authors declare that they have no competing interests.

Received: 1 October 2015 Accepted: 3 January 2016

Published online: 15 January 2016

\section{References}

1. Oregon Seed Association. http://www.oregonseed.org/ (2014). Accessed 25 Sept 2015.

2. Young W. Crop and soil science, Oregon State University. http://cropandsoil.oregonstate.edu/content/oregon-grass-and-legume-seed-production (2014). Accessed 25 Sept 2015.

3. Alderman S, Coats D, Crowe F. Impact of Ergot on Kentucky bluegrass grown for seed in Northeastern Oregon. Plant Dis. 1996;80:853-5.

4. Pažoutová S. Evolutionary strategy of Claviceps. In: White JF, Bacon CW, Hywel-Jones NL, Spatafora JW, editors. Clavicipitalean Fungi: evolutionary biology, chemistry, biocontrol and cultural impacts. New York: Marcel Dekker; 2003. p. 329-54.

5. Schumann GL. Ergot. Plant Health Inst. 2000. doi:10.1094/ PHI-I-2000-1016-01.

6. Miedaner T, Geiger H. Biology, Genetics, and Management of ergot (Claviceps spp.) in rye, sorghum, and pearl millet. Toxins. 2015;7:659-78. doi:10.3390/toxins7030659.

7. Randhawa H, Asif M, Pozniak C, Clarke J, Graf R, Fox S, Humphreys G, Knox R, DePauw R, Singh A, Cuthbert R, Hucl P, Spaner D. Application of molecular markers to wheat breeding in Canada. Review. Plant Breeding. 2013;132:458-71.

8. Parh D, Jordan D, Aitken E, McIntyre L, Godwin I. Sorghum ergot: revealing the genetic architecture of resistance. Proceedings of the 4th International Crop Sciences Congress (1-4). 2004.

9. Jungehülsing U, Tudzynski P. Analysis of genetic diversity in Claviceps purpurea by RAPD markers. Mycol Res. 1997;101(1):1-6.

10. Pažoutová S, Cagaš B, Kolínská R, Honzátko A. Host Specialization of different populations of ergot fungus (Claviceps purpurea). Czech J. Genet. Plant Breeding. 2002;38(2):75-81.

11. Pažoutová S, Olšovská J, Linka M, Kolínská R, Flieger M. Chemoraces and habitat specialization of Claviceps purpurea populations. Appl Environ Microbiol. 2000;66(12):5419-25.

12. Pažoutová S, Raybould A, Honzátko A, Kolínská R. Specialized population of Claviceps purpurea from salt marshes. Mycol Res. 2002;106(2):210-4.
13. Negård M, Uhlig S, Kauserud H, Andersen T, Høiland K, Vrålstad T. Links between Genetic Groups, Indole alkaloid profiles and ecology within the grass-parasitic Claviceps purpurea species complex. Toxins. 2015;7(5):1431-56.

14. Pažoutová S, Pešicová K, Chudíčková M, Šrůtka P, Kolařík M. Delimitation of cryptic species inside Claviceps purpurea. Fungal Biol. 2015;2015(119):7-26. doi:10.1016/j.funbio.2014.10.003.

15. Fisher AJ, Gordon TR, Ditomaso JM. Geographic distribution and diversity in Claviceps purpurea from salt marsh habitats and characterization of Pacific coast populations. Mycol Res. 2005;109:439-46.

16. Douhan G, Smith M, Huyrn K, Westbrook A, Beerli P, Fisher A. Multigene analysis suggests ecological speciation in fungal pathogen Claviceps purpurea. Mol Ecol. 2008;17(9):2276-86.

17. Oeser B, Beaussart F, Haarmann T, Lorenz N, Nathues E, Rolke Y, Scheffer J, Weiner J, Tudzynski P. Expressed sequence tags from the flower pathogen Claviceps purpurea. Mol Plant Path. 2009;10(5):665-84.

18. Schardl CL, Young C, Hesse U, Amyotte S, Andreeva K, Calie P, Fleetwood D, Haws D, Moore N, Oeser B, Panaccione D, Schweri K, Voisey C, Farman M, Jaromczyk J, Roe B, O'Sullivan D, Scott B, Tudzynski P, An Z, Arnaoudova E, Bullock C, Charlton N, Chen L, Cox M, Dinkins R, Florea S, Glenn A, Gordon A, Güldener U, Harris D, Hollin W, Jaromczyk J, Johnson R, Khan A, Leistner E, Leuchtmann A, Li C, Liu J, Liu M, Mace W, Machado C, Nagabhyru P, Pan J, Schmid J, Sugawara K, Steiner U, Takach J, Tanaka E, Webb J, Wilson E, Wiseman J, Yoshida R, Zeng Z. Plant-symbiotic fungi as chemical engineers: multi-genome analysis of the Clavicipitaceae reveals dynamics of alkaloid loci. PLoS Genet. 2013;9(2):e1003323. doi:10.1371/ journal.pgen.1003323.

19. Powell W, Machray G, Provan J. Polymorphism revealed by simple sequence repeats. Trends Plant Sci. 1996;1:215-22.

20. Scott J, Kaur N, Alderman S, Walenta D, Hamm P, Frost K, Dung J. Molecular differentiation of Claviceps isolates from Kentucky bluegrass and perennial ryegrass in Oregon and Washington. Poster session presented at: epidemiology-population biology genetics. APS Annual Meeting, Pasadena, CA; 2015.

21. National Center for Biotechnology Information. NCBI BioSample number: SAMEA2272775. http://www.ncbi.nlm.nih.gov/bioproject/PRJEA76493/ (2015). Accessed 25 Sept 2015.

22. Dutech C, Eknjalbert J, Fournier E, Delmotte F, Barrès B, Carlier J, Tharreau $D$, Giraud T. Challenges of microsatellite isolation in fungi. Fungal Genet Biol. 2007;44:933-49.

23. Gilmore B, Hummer $K$, Bassil N. DNA extraction protocols from dormant buds of twelve woody plant genera. J. Amer. Pomol. Soc. 2011;65:201-7.

24. Faircloth BC. MSATCOMMANDER: detection of microsatellite repeat arrays and automated, locus-specific primer design. Mol Ecol Resources. 2015;2008(8):92-4. doi:10.1111/j.1471-8286.2007.01884.x.

25. Rozen S, Skaletsky HJ. Primer3 on the WWW for general users and for biologist programmers. In: Krawetz S, Misener S, editors. Bioinformatics Methods and Protocols: Methods in Mol Bio. Totowa: Humana Press; 2000

26. Koressaar T, Remm M. Enhancements and modifications of primer design program Primer3. Bioinformatics. 2007;23(10):1289-91.

27. Untergrasser A, Cutcutache T, Koressaar T, Ye J, Faircloth B, Remm M, Rozen S. Primer3-new capabilities and interfaces. Nucleic Acids Res. 2012;40(15):e115.

28. Schuelke M. An economic method for the fluorescent labeling of PCR fragments. Nat Biotechnol. 2000;18:233-4.

29. Brownstein MJ, Carpten JD, Smith JR. Modulation of non-templated nucleotide addition by Taq DNA polymerase: primer modifications that facilitate genotyping. Biotechniques. 1996;20(6):1004-6 (1008-10).

30. Jombart T. Adegenet: a R package for the multivariate analysis of genetic markers. Bioinformatics. 2015;2008(24):1403-5. doi:10.1093/ bioinformatics/btn 129 .

31. Jombart T, Devillard S, Balloux F. Discriminant analysis of principal components: a new method for the analysis of genetically structured populations. BMC Genetics. 2010;11:94. doi:10.1186/1471-2156-11-94.

32. Jombart T, Ahmed I. Adegenet 1.3-1: new tools for the analysis of genome-wide SNP data. Bioinformatics. 2011;27(21):3070-1. doi: 10.1093/ bioinformatics/btr521.

33. R Development Core Team, R version 3.2.1 "World-Famous Astronaut." R: A Language and Environment for Statistical Computing. Vienna: R Foundation for Statistical Computing. http://www.R-project.org/ (2015). Accessed 25 Sept 2015. 
34. Kamvar ZN, Tabima JF, Grünwald NJ. Poppr: an R package for genetic analysis of populations with clonal, partially clonal, and/or sexual reproduction. PeerJ. 2014;2:e281. doi:10.7717/peerj.281.

35. Bruvo R, Michiels NK, D'Souza TG, Schulenburg H. A simple method for the calculation of microsatellite genotype distances irrespective of ploidy level. Mol Ecol. 2004;13(7):2101-6.

36. Tamura K, Dudley J, Nei M, Kumar S. MEGA4: Molecular Evolutionary Genetics Analysis (MEGA) software version 4.0. Mol Biol Evol. 2007:24:1596-9.
37. Liu K, Muse S. PowerMarker: an integrated analysis environment for genetic marker analysis. Bioinformatics. 2005;21:2128-9.

38. Spellerberg I, Fedor P. A tribute to Claude Shannon (1916-2001) and a plea for more rigorous use of species richness, species diversity and the 'Shannon-Wiener' index. Glob Ecol Biogeogr. 2003;12:177-9.
Submit your next manuscript to BioMed Central and we will help you at every step:

- We accept pre-submission inquiries

- Our selector tool helps you to find the most relevant journal

- We provide round the clock customer support

- Convenient online submission

- Thorough peer review

- Inclusion in PubMed and all major indexing services

- Maximum visibility for your research

Submit your manuscript at www.biomedcentral.com/submit
(O) BioMed Central 